\title{
Appendicite à gauche révélant un mésentère commun
}

\section{Left-Sided Appendicitis Revealing a Common Mesentery}

\section{E. Robeley - T. Desmettre}

Reçu le 13 octobre 2017; accepté le 26 décembre 2017

(C) SFMU et Lavoisier SAS 2018

\section{Introduction}

L'appendicite aiguë est la plus fréquente des urgences chirurgicales abdominales et représente en France $30 \%$ des interventions abdominales [1]. Son diagnostic peut être porté tardivement devant des symptômes atypiques ou peu inquiétants. L'appendicite aiguë révélant un mésentère commun complet est un diagnostic rare chez l'adulte [2]. Le cas clinique rapporté met en exergue les difficultés diagnostiques de cette affection.

\section{Observation}

Une patiente de 20 ans consulte deux fois dans la même journée pour des douleurs abdominales, évoluant depuis cinq jours, non soulagées par différentes classes d'antalgiques et d'antispasmodiques. Il s'agit de douleurs fluctuantes, en barre, sous-ombilicales à type de coliques. Parmi ses antécédents, on note un vitiligo, une hypothyroïdie et une cataracte congénitale. À l'examen, la tension artérielle est à $132 / 79 \mathrm{mmHg}$, la fréquence cardiaque à $77 \mathrm{c} / \mathrm{min}$, la saturation en oxygène périphérique à $100 \%$ en air ambiant, la température à $36,4^{\circ} \mathrm{C}$, et l'échelle numérique de la douleur est à 6 . La bandelette urinaire est négative. L'abdomen est sensible à la palpation périombilicale ainsi qu'au flanc gauche sans défense. La biologie retrouve une CRP à $10,8 \mathrm{mg} / \mathrm{l}$ (normale $<5 \mathrm{mg} / \mathrm{l}$ ), les leucocytes sont à $10300 / \mathrm{mm}^{3}$ (norme de 3800 à $9500 / \mathrm{mm}^{3}$ ), la procalcitonine et les $\beta H C G$ sont négatives. Le bilan hépatopancréatique ainsi que l'ionogramme sont normaux. Devant la régression totale

\section{E. Robeley $(\bowtie) \cdot T$. Desmettre}

Service d'accueil des urgences/SAMU 25, CHRU de Jean-Minjoz, 1, boulevard Fleming, F-25030 Besançon cedex, France

e-mail : elise.robeley@wanadoo.fr

T. Desmettre

UFR SMP, université de Franche-Comté, rue Ambroise-Paré, F-25030 Besançon cedex, France des douleurs, elle regagne son domicile. Elle se présente à nouveau aux urgences le lendemain. La patiente est apyrétique, algique (échelle numérique à 5) ; les paramètres vitaux sont normaux. La bandelette urinaire est négative. Les douleurs siègent dans le flanc gauche, l'examen clinique reste inchangé. La patiente ne présente ni nausées ni vomissements, il n'y a pas d'arrêt des matières et des gaz. La biologie retrouve cette fois une CRP à $27,5 \mathrm{mg} / 1$, les leucocytes sont à $9300 / \mathrm{mm}^{3}$. Le bilan hépatopancréatique est normal. L'échographie abdominopelvienne est sans particularité, mais l'appendice et l'ovaire gauche ne sont pas visualisés, et dans ce contexte, un complément d'imagerie est demandé. Le scanner abdominopelvien injecté retrouve une appendicite aiguë, non compliquée, en position ascendante en fosse iliaque gauche sur mésentère commun complet (Fig. 1). L'exploration cœlioscopique a confirmé le diagnostic de malrotation avec appendicite aiguë. L'évolution après l'appendicectomie a été marquée au septième jour par un syndrome occlusif d'origine mécanique, nécessitant une laparotomie en urgence avec mise en évidence de brides localisées au niveau du cæcum, responsables de l'obstruction des deux dernières anses du grêle.

\section{Discussion}

Au cours du développement embryonnaire, l'intestin subit des phénomènes de réintégration, de rotation et d'accolement. Lorsque ces phénomènes sont incomplets ou absents, ils sont à l'origine de malpositions du tube digestif pouvant entrainer des complications digestives. Ces malformations peuvent toucher une naissance sur 500 [3]. Elles demeurent très souvent asymptomatiques et donc non diagnostiquées. Il existe plusieurs anomalies de rotation de l'anse intestinale, qui vont de l'absence totale de rotation à l'arrêt de rotation à $90^{\circ}$ ou $180^{\circ}$. Le mésentère commun complet rencontré dans cette présentation correspond à un arrêt de la rotation à $90^{\circ}$. Il se traduit par la position de l'ensemble des anses grêles dans la partie droite de l'abdomen, tandis que le côlon se situe en totalité dans la partie gauche. Cette malposition n'est 

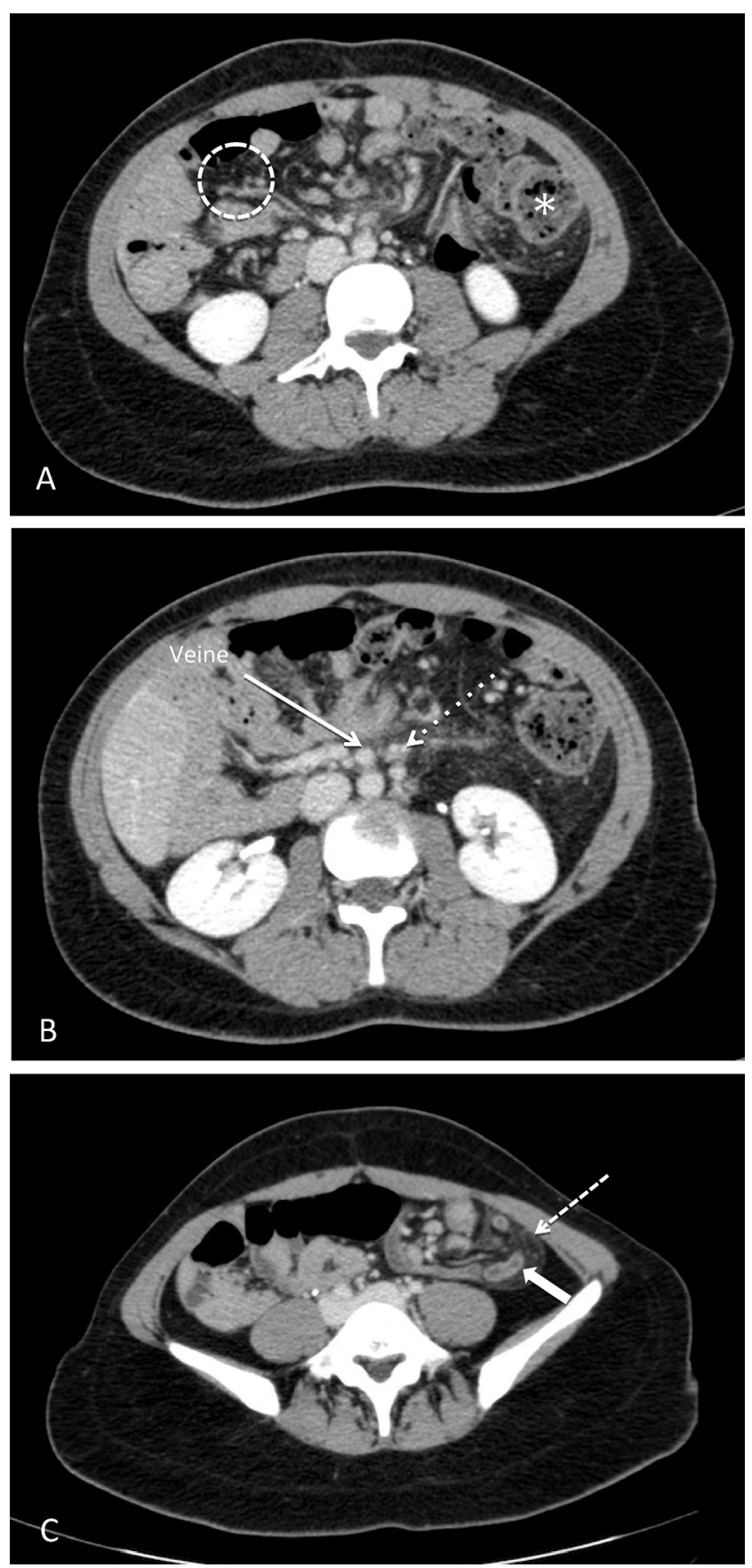

Fig. 1 Tomodensitométrie abdominopelvienne en coupe axiale. A. Visualisation du grêle à droite (entouré par les pointillés) et du côlon droit à gauche (étoile). B. Inversion position artère et veine mésentérique supérieure (flèche pleine : artère mésentérique, flèche pointillée : veine mésentérique). C. Appendicite aiguë, non compliquée, en position ascendante en fosse iliaque gauche (flèche blanche large) sur mésentère commun avec infiltration de la graisse (flèche tiret)

pas pathogène et reste asymptomatique. Dans notre observation, le côlon est localisé uniquement dans la partie gauche de l'abdomen, les anses grêles à droite, l'angle de Treitz est absent, et la veine mésentérique supérieure est située à gauche de l'artère mésentérique supérieure (Fig. 1AB).

Une autre présentation possible correspond au mésentère commun incomplet qui correspond à un arrêt de la rotation à $180^{\circ}$, le grêle se plaçant à gauche du côlon. Cette situation est à très haut risque de volvulus du grêle et d'infarctus intestinomésentérique, puisque seuls $15 \%$ des mésentères communs incomplets restent asymptomatiques, contrairement au mésentère commun complet qui n'expose pas au risque de volvulus.

La localisation à gauche de l'appendice (Fig. 1C) est extrêmement rare $[2,4]$. Une revue de la littérature rapporte 63 cas qui s'inscrivent dans le cadre d'un situs inversus $[5,6]$; les cas en rapport avec un mésentère commun complet sont encore plus rares $[3,7]$. Ces présentations atypiques sont donc responsables d'un retard diagnostique pouvant être à l'origine de complications graves. À l'heure actuelle, le diagnostic se fait de plus en plus précocement, grâce à l'échodoppler et surtout à la tomodensitométrie $[8,9]$.

En conclusion, certaines variabilités anatomiques rares sont responsables de présentations atypiques d'appendicites à gauche.

Liens d'intérêts Les auteurs déclarent ne pas avoir de liens d'intérêts.

\section{Références}

1. Flamant Y; Associations de recherche en chirurgie (2005) Douleurs abdominales aiguës de l'adulte. In: Rambaud JC (ed) Traité de gastro-entérologie. Flammarion, Paris, pp 198-210

2. Pinto A, Raimondo DD, Tuttolomondo A, et al (2007) An atypical clinical presentation of acute appendicitis in a young man with midgut malrotation. Radiography 13:164-8

3. Welte FJ, Grosso M (2007) Left-sided appenditis in a patient with congenital gastrointestinal malrotation: a case report. J Med Case Rep 1:92

4. Nelson MJ, Pesola GR (2001) Left lower quadrant pain of unusual cause. J Emerg Med 20:241-5

5. Akbulut S, Caliskan A, Ekin A, Yagmur Y (2010) Left-sided acute appendicitis with situs inversus totalis: review of 63 published cases and report of two cases. J Gastrointest Surg 14:1422-8

6. Huang SM, Yao CC, Tsai TP, Hsu GW (2008) Acute appendicitis in situs inversus totalis. J Am Coll Surg 207:954

7. Flesch J, Oswald P, Grebici M, et al (2010) Mésentère commun complet révélé par une appendicite perforée gauche. J Radiol 91:915-6

8. Israelit S, Brook OR, Nira BR, et al (2009) Left-sided perforated acute appendicitis in an adult with midgut malrotation: the role of computed tomography. Emerg Radiol 16:217-8

9. Perera WR, Hennessy OF (2010) Clinical images - an unusual case of appendicitis. Am J Surg 199:e79-e81 